\title{
Multisystem inflammatory syndrome in neonates (MIS-N) associated with SARS-CoV2 infection: a case series
}

\author{
Kiran More ${ }^{1}$ (]) Sheila Aiyer ${ }^{2} \cdot$ Ashish Goti $^{3} \cdot$ Manan Parikh $^{4} \cdot$ Samir Sheikh $^{5} \cdot$ Gaurav Patel $^{6} \cdot$ Venkat Kallem $^{7}$. \\ Roopali Soni ${ }^{1,8} \cdot$ Praveen Kumar $^{9}$
}

Received: 26 August 2021 / Revised: 5 January 2022 / Accepted: 6 January 2022 / Published online: 14 January 2022

(c) The Author(s), under exclusive licence to Springer-Verlag GmbH Germany, part of Springer Nature 2022

\begin{abstract}
Multisystem inflammatory syndrome in neonates (MIS-N) is hypothesised to be caused either following transplacental transfer of SARS-CoV2 antibodies or antibodies developed in the neonate after infection with SARS-CoV-2. In this paper, we aim to discuss the clinical manifestations, laboratory features, and management of neonates diagnosed with MIS-N. We collated information from five participating hospitals in western India. A cohort of newborn infants presenting with multi-system involvement, along with the presence of SARS-CoV2 antibodies, was identified. Current proposed international diagnostic criteria for MIS-N were used to group the cases into three categories of Most likely, Possible, and Unlikely MIS-N. A total of 20 cases were reported with a diagnosis of MIS-N, all having high titres of SARS CoV2 IgG antibodies and negative for SARS CoV2 antigens. Most likely $\operatorname{MIS}(n=5)$ cases presented with respiratory distress (4/5), hypotension and shock (4/5), and encephalopathy (2/5). Inflammatory markers like CRP (1/5), Procalcitonin (1/5), Ferritin (3/5), D-dimer (4/5), and LDH (2/5) were found to be elevated, and four of them had significantly high levels of proBNP. The majority of them (4/5) responded to immunomodulators, three neonates were discharged home, and two died. Possible MIS infants $(n=9)$ presented with fever (7/9), respiratory distress (4/9), refusal to feed (6/9), lethargy (5/9), and tachycardia (3/9). ProBNP as a marker of cardiac dysfunction was noted to be elevated in four (4/9) infants, correlating with abnormal echocardiography findings in two. In the Unlikely MIS $(n=6)$ category, three $(3 / 6)$ infants presented with respiratory distress, one (1/6) with shock and cardiac dysfunction, and only one (1/6) with fever. All of them had elevated inflammatory markers. However, there were other potential diagnoses that could have been responsible for the clinical scenarios in these six cases.

Conclusion: MIS-N requires a high index of suspicion and should be considered in a neonate presenting with two or more systems involvement, in the presence of SARS-CoV2 antibodies, along with elevated inflammatory markers, once other common neonatal conditions have been ruled out.
\end{abstract}

\section{What is Known:}

- Severe acute respiratory syndrome coronavirus-2 (SARS-CoV2) associated multisystem inflammatory syndrome in children (MIS-C) is widely reported in paediatric population, however only few reports of newborn affection.

- MIS-C is known to cause by virus-induced post-infective antibody mediated immune dysregulation with severe multi-system affection.

What is New:

- MIS-N may present with varied clinical manifestations with multi-system involvement of variable severity with milder disease in term and severe disease with cardiac dysfunction in preterm newborns.

- Multisystem inflammatory syndrome in newborns $(M I S-N)$ is postulated to occur following immune dysregulation associated with transplacental transfer of SARS-CoV2 antibodies or antibodies developed in the neonate after infection with SARS-CoV-2.

Keywords Multisystem inflammatory syndrome in neonates $\cdot$ Severe acute respiratory syndrome coronavirus-2 (SARSCoV2) · Coronavirus Disease 2019 (COVID-19) · Reverse transcriptase-polymerase chain reaction (RT-PCR) · SARSCoV2 immunoglobulins

\section{Communicated by Daniele De Luca}

Kiran More

kmore@sidra.org

Extended author information available on the last page of the article

$\begin{array}{ll}\text { Abbreviations } \\ \text { ANC } & \text { Absolute neutrophil count } \\ \text { COVID-19 } & \text { Coronavirus Disease 2019 } \\ \text { CDC } & \text { Centres for Disease Control } \\ \text { CRP } & \text { C-reactive protein }\end{array}$

\section{Abbreviations}

ANC

CDC

CRP
Absolute neutrophil count
Centres for Disease Control
-reactive protein




\begin{tabular}{|c|c|}
\hline ESR & Erythrocyte sedimentation rate \\
\hline IL-6 & Interleukin 6 \\
\hline IG & Immunoglobulins \\
\hline LDH & Lactate dehydrogenase \\
\hline LMWH & Low molecular weight heparin \\
\hline MIS-N & $\begin{array}{l}\text { Multisystem inflammatory syndrome in } \\
\text { neonates }\end{array}$ \\
\hline PCT & Procalcitonin \\
\hline Pro-BNNP & Pro B-type natriuretic peptide \\
\hline RT-PCR & $\begin{array}{l}\text { Reverse transcriptase-polymerase chain } \\
\text { reaction }\end{array}$ \\
\hline SARS-CoV2 & $\begin{array}{l}\text { Severe acute respiratory syndrome } \\
\text { coronavirus- } 2\end{array}$ \\
\hline TLC & Total Leucocyte count \\
\hline
\end{tabular}

\section{Introduction}

Severe acute respiratory syndrome coronavirus-2 (SARS$\mathrm{CoV} 2$ ) associated with multisystem inflammatory syndrome in children (MIS-C) has been increasingly reported worldwide with the spread of Coronavirus Disease 2019 (COVID19) infection $[1,2]$. The exact pathogenesis for MIS-C remains elusive. Virus-induced post-infective immune dysregulation appears to play a predominant role [3]. The neonatal immune system is immature and may not produce sufficient SARS-CoV-2 antibodies. However, a large study cohort has recently demonstrated efficient transplacental transfer of immunoglobulins (IG-G) antibodies from mother to fetus [4]. These immunoglobulins are generally thought to be protective against SARS-CoV-2 infection, but the transplacental transfer of immunoglobulins along with inutero transfer of other inflammatory cytokines may mimic a process similar to MIS-C, potentially causing immune activation and manifesting as a multisystem inflammatory syndrome in neonates (MIS-N) [5]. Seroconversion response to SARS-CoV-2 infection has been seen within 2-3 weeks of symptomatic infection, with both immunoglobulin-M (IGM) and IG-G levels detected in plasma [6, 7]. Newborns acquiring perinatal SARS-CoV-2 can also mount an antibody response, and there is the possibility that there may be a late manifestation with a presentation similar to MIS-C seen in children [8]. This condition has been reported anecdotally in the newborn population, presenting with fever and multi-site inflammation, especially myocarditis manifesting clinically or with raised biomarkers such as Troponin and ProB-type natriuretic peptide(proBNP) [9].

MIS-C in the paediatric population is now better described; however, the spectrum of possible manifestations in neonates presenting with MIS-N has only been reported in a small number of cases [5, 10-16]. Authors of this case series (KM and PK) were involved in formally collecting data for the national Indian registry of SARS-CoV2 in newborns, up to August 2020 which was presented at national neonatal conferences and subsequently published [17]. MIS-N as a clinical condition was unrecognised at this stage and therefore not suspected by clinicians in this particular cohort. Later, as the pandemic evolved, some tertiary centres from Western India contacted the authors to report on newborn infants presenting with clinical features suggestive of MIS-N syndrome. In this article, we describe clinical features, variety of presentations, and diagnostic criteria for MIS-N in a cohort of neonates exposed to SARS-CoV-2 antenatally, perinatally, and postnatally.

\section{Methods}

Cases reported by clinicians from five tertiary centres from Western India, between October 2020 and March 2021, were collated and analysed. Treating clinicians followed clinical protocols and management guidelines published by joint recommendation from the Federation of Obstetric and Gynaecological Societies of India (FOGSI), National Neonatology Forum of India (NNF), and Indian Academy of Paediatrics (IAP) $[18,19]$. These guidelines were adapted from Indian Council of Medical Research (ICMR) protocol June 2020 which were based on the World Health Organisation (WHO)/Centre for Disease Control (CDC)guidelines (see Appendix). SARS-CoV2 antigens were tested using the realtime polymerase chain reaction (RTPCR) method in nasopharyngeal swabs, whereas SARS-CoV2 immunoglobulins were tested on Roche Cobas e411 by ECLIA method in both maternal and neonatal serum samples. This method detects total antibodies against SARS-CoV-2, and levels $>1.00 \mathrm{~S} /$ $\mathrm{CO}$ index were considered reactive or positive. In all cases, the baseline investigations included complete blood count (CBC), C-reactive protein (CRP), procalcitonin (PCT), D-dimer, Creatinine Kinase (CK-MB), or Troponin (TropT), and coagulation profile. In addition, Ferritin and Interleukin (IL6) levels were monitored when a cytokine storm was suspected. In cases with suspected cardiac involvement, lactate dehydrogenase (LDH), Troponin, and Pro B-type natriuretic peptide(proBNP) were done to assess myocardial involvement/dysfunction, and also echocardiography (ECHO) was performed as and when feasible. Immunomodulator therapy was based on the above guidelines and recommendations with choices and doses used as described in Appendix.

\section{Inclusion criteria}

There are defined clinical criteria for MIS-C in children [20]; however, for newborns, there is no clear agreed definition of MIS-N. Therefore, we decided to extrapolate the definition of MIS-N from CDC criteria, as used in a similar 
case series, [5] along with published definitions for neonatal SARS-CoV-2 infection, [21, 22] and agreed upon certain inclusion criteria.

Once common neonatal conditions mimicking multisystem presentations (e.g. sepsis, birth asphyxia, pneumonia, or any other common condition) were ruled out by the responsible clinician, MIS-N was considered in neonates meeting the following criteria:

(i) Symptoms presenting from birth to the first four weeks of life

(ii) Fever along with two or more systems involvement (fever, if present, is suggestive but not mandatory for diagnosis in the newborn)

(iii) Evidence of raised inflammatory markers such as CRP, PCT, D Dimer, Ferritin, and IL-6

(iv) Evidence of SARS-CoV-2 antibodies in the neonate (considered mandatory for a diagnosis of MIS) and SARS-CoV-2 antigen should be negative during the presentation to rule out active SARS-CoV-2 infection to support the immunological process

\section{AND}

Associated evidence of maternal SARS-CoV-2 infection, defined as either supporting history or laboratory (positive SARS-CoV-2 quantitative RT-PCR test in a nasopharyngeal sample during the peripartum period) or epidemiological evidence of infection in the form of SARS-CoV-2 antibodies (for confirming the transplacental mechanism of MIS-N)

OR

History of prior confirmed neonatal SARS-CoV-2 infection (for confirming mechanism of MIS-N due autoantibodies secondary to SARS-CoV-2 infection) which can be classified based on the type of SARS-CoV-2 transmission in neonates as per WHO criteria as follows-

(a) In utero transmission - nasopharyngeal swab at age $<24 h$ positive for SARS-CoV-2 and positive serology for SARS-CoV-2 IGM and IG G. (b) Intrapartum transmission - at least one test obtained at age $<24 \mathrm{~h}$ negative for SARS-CoV-2 with Negative serology (IgM or IgA) followed by positive RT-PCR at $24-48 h$ or positive serology (IgM or $\operatorname{Ig} \mathrm{A})$ at age 7-14 days that is corroborated by a positive serology test on a second sample obtained within 10 days of the first positive test. (c) Postpartum transmission - at least one test obtained at age $<48 \mathrm{~h}$ was performed and negative for SARS-CoV-2 with Negative serology (IgM or IgA) at age $<14$ days followed by positive RT-PCR at age $>48$ $h$ OR positive serology (IgM or IgA) at age $>14$ days that is corroborated by a second positive serology test obtained within 10 days of the first positive test at age $>14$ days.
Based on our inclusion criteria, we classified cases into the following categories:

a) Most likely MIS — where the clinical picture meets all defined criteria.

b) Possible MIS - where there is an unusual clinical presentation, suspicious of MIS, but may not meet all specified criteria but no other alternative diagnosis was indicated. The presence of fever in newborns is considered unusual so fever with one more system affection along with supportive biochemical markers was also considered as possible MIS.

c) Unlikely MIS - where there is an unusual clinical presentation, suspicious of MIS, but does not meet all specified criteria and there was associated possible alternative diagnosis.

\section{Exclusion criteria}

Those cases where SARS-CoV-2 antibodies were present but the clinical presentation was neither unusual nor of a multisystem nature were excluded.

We also subdivided the MISN cases based on the timing of presentation, to differentiate the possible source of SARSCoV-2 antibodies:

(1) Early MISN (within first $72 \mathrm{~h}$ of life) - hypothesised as presenting due to transplacental transfer of maternal SARS-CoV-2 antibodies.

(2) Late MISN (beyond $72 \mathrm{~h}$ of life) - hypothesised as occurring either due to antibodies produced secondary to SARS-CoV-2 infection in the newborn or transplacental transfer of maternal SARS-CoV-2 antibodies (depending on lag period needed for antibody production)

The following data was collected for all reported cases:

(a) Demographics: birth gestation, weight, etc. along with maternal history for SARS-CoV-2 infection

(b) Serology: maternal and neonatal Serology for SARS$\mathrm{CoV}-2$ - both antigen and antibodies to ascertain the mode of transmission

(c) Clinical features and laboratory results

(d) Management and outcomes

Age-specific normal ranges were used for interpreting laboratory parameters. Abnormal values were defined as serum ferritin values of $>260 \mathrm{ng} / \mathrm{ml}$ in term newborn and $>200 \mathrm{ng} / \mathrm{ml}$ in preterm [23], D-dimer $>200 \mathrm{ng} / \mathrm{ml}$, Pro B-type natriuretic peptide (proBNP) $>700 \mathrm{pg} / \mathrm{ml}$, and serum lactate dehydrogenase $>450 \mathrm{U} / 1$ [24]. Where indicated, echocardiogram (ECHO) was performed by a paediatric cardiologist to assess cardiac function and to look for abnormal coronary dilatation. Cardiac dysfunction or myocarditis on ECHO was defined as decreased 
left ventricular (LV) ejection fraction $(\mathrm{EF})<55 \%$ and fractional shortening (FS) $<26 \%$ on functional echocardiography [25].

Parental consent was obtained from each case, and permission from local hospital authorities was obtained for data sharing. Ethics committee approval was obtained from the primary site. We followed the CARE guidelines [26] for reporting this case series, and a checklist for the same is provided as a supplement.

\section{Summary of cases}

A total of 26 neonates were reported to be diagnosed and treated for MIS-N. We analysed all 26 cases. Six were excluded as they had positive antibodies but no multisystem affection. Twenty cases were therefore analysed as shown in case selection flow diagram (Fig. 1).

Table 1 summarises the clinical characteristics and management of all 20 cases.

\section{Clinical findings}

Most likely MIS $(n=5)$ The majority (4/5) presented within the first week of life, with two neonates presenting on day 1 , one on day 2 , and another on day 5 . One presented late, on day 30 of life. Figure 2 summarises the clinical and laboratory profile of all cases in this group. The most common clinical findings noted in these neonates were two system involvement, i.e. respiratory distress (4/5) and shock with hypotension (4/5), whereas two babies in addition, also had encephalopathy (2/5). Fever and seizures were noted only in one neonate. Most likely cases are summarised in Fig. 2.

Possible MIS $(n=9)$ Of the nine infants, four presented within the first week of life and the remaining five from the second to the fourth week. The most common clinical findings noted in these infants were fever (7/9), respiratory distress (4/9), refusal of feeds (6/9), lethargy (5/9), and tachycardia (3/9). One infant (case 6) had severe multi-system manifestation; however, SARS-CoV-2 IG-M was positive with negative SARS-CoV-2 RTPCR, so intrauterine or perinatally acquired SARS-CoV-2 infection could not be ruled out.

Unlikely MIS $(n=6)$ Category, three (3/6) infants presented with respiratory distress, one (1/6) with shock and cardiac dysfunction, and one (1/6) with fever. All the neonates in this category had a strong alternative diagnosis in the form of sepsis, respiratory distress syndrome, necrotising enterocolitis, and parotitis, which could also explain the clinical presentation in the infant.
Fig. 1 Flow diagram of case selection and distribution
Figure 1: Flow Diagram of Cases selection and distribution

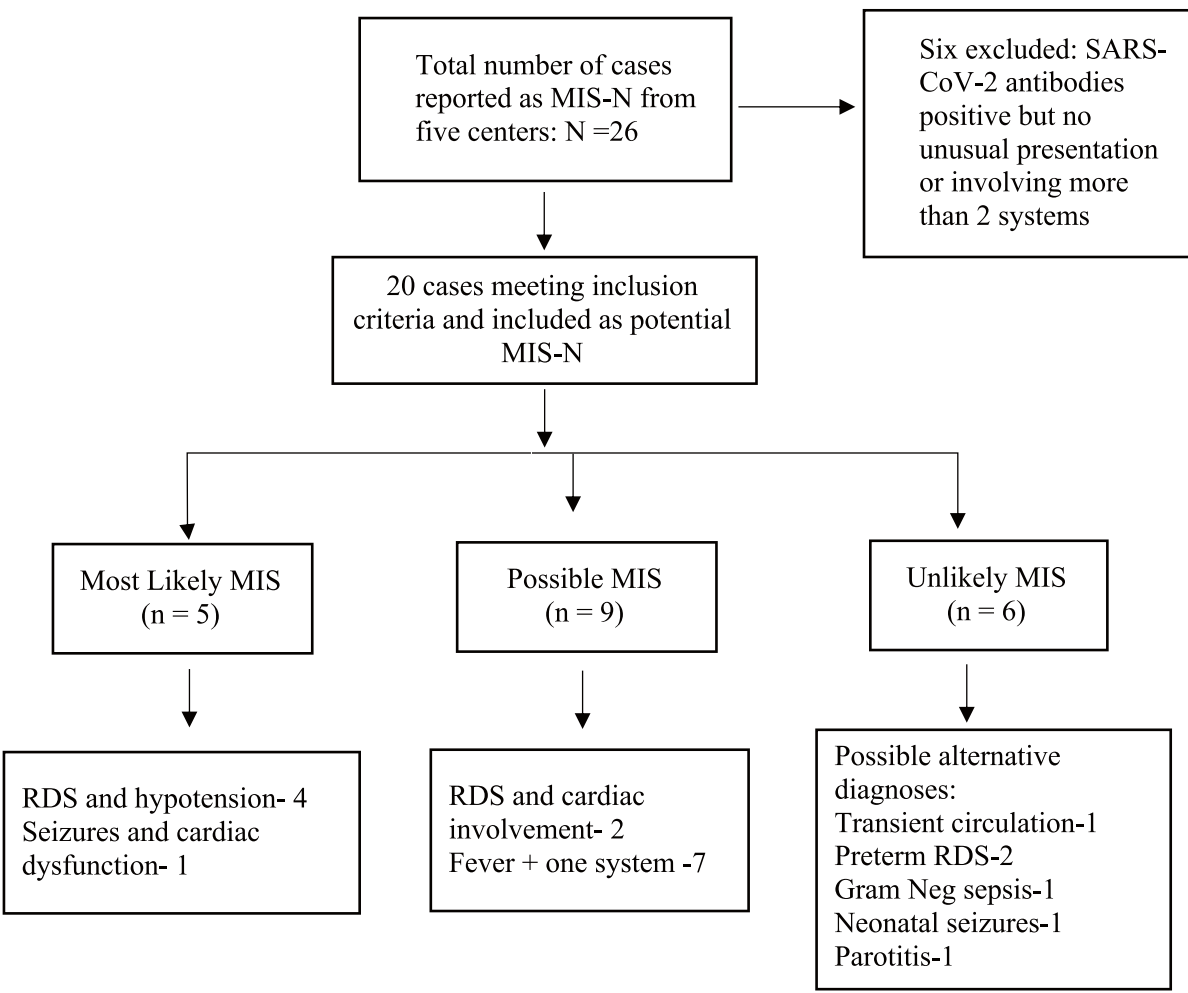




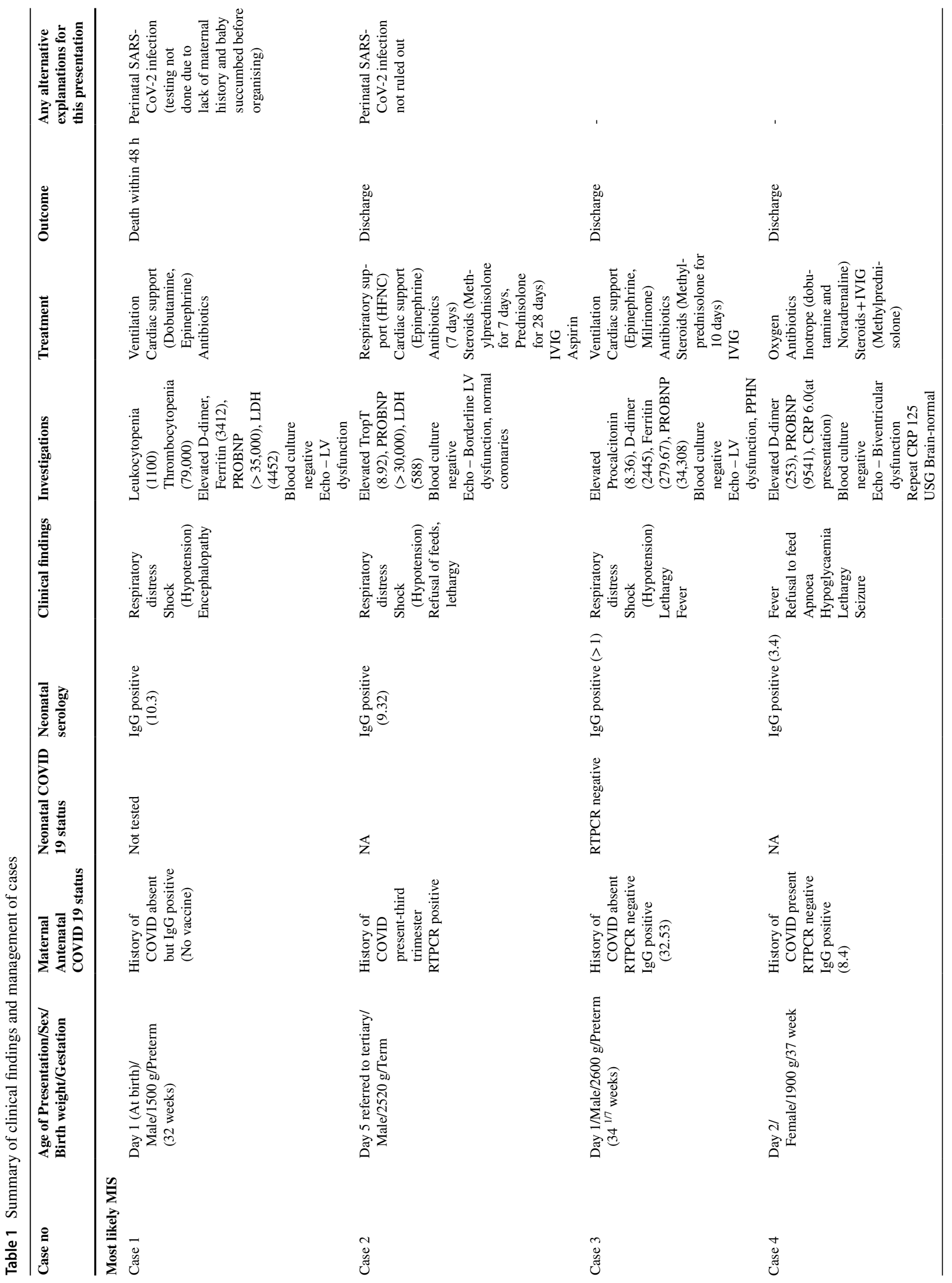




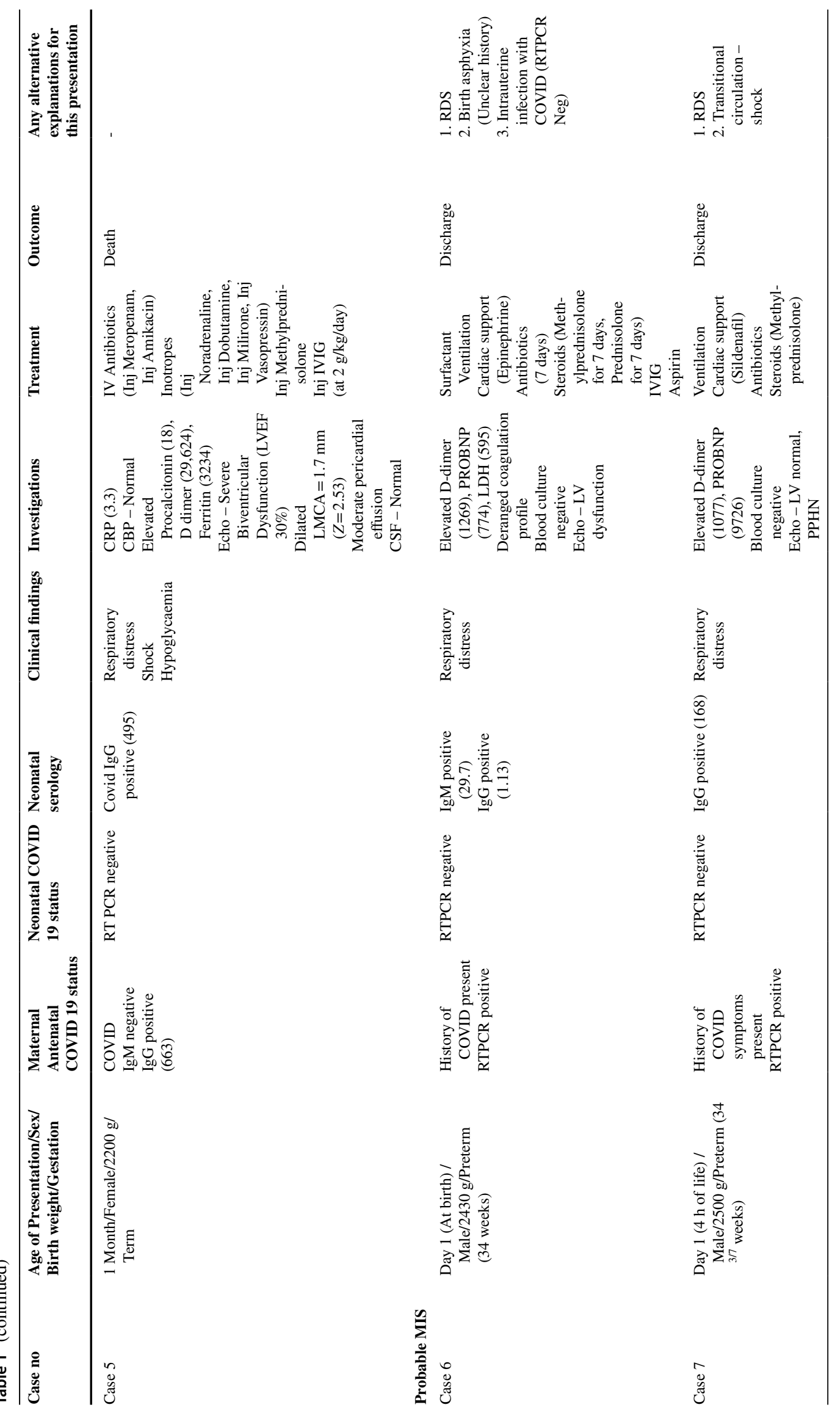




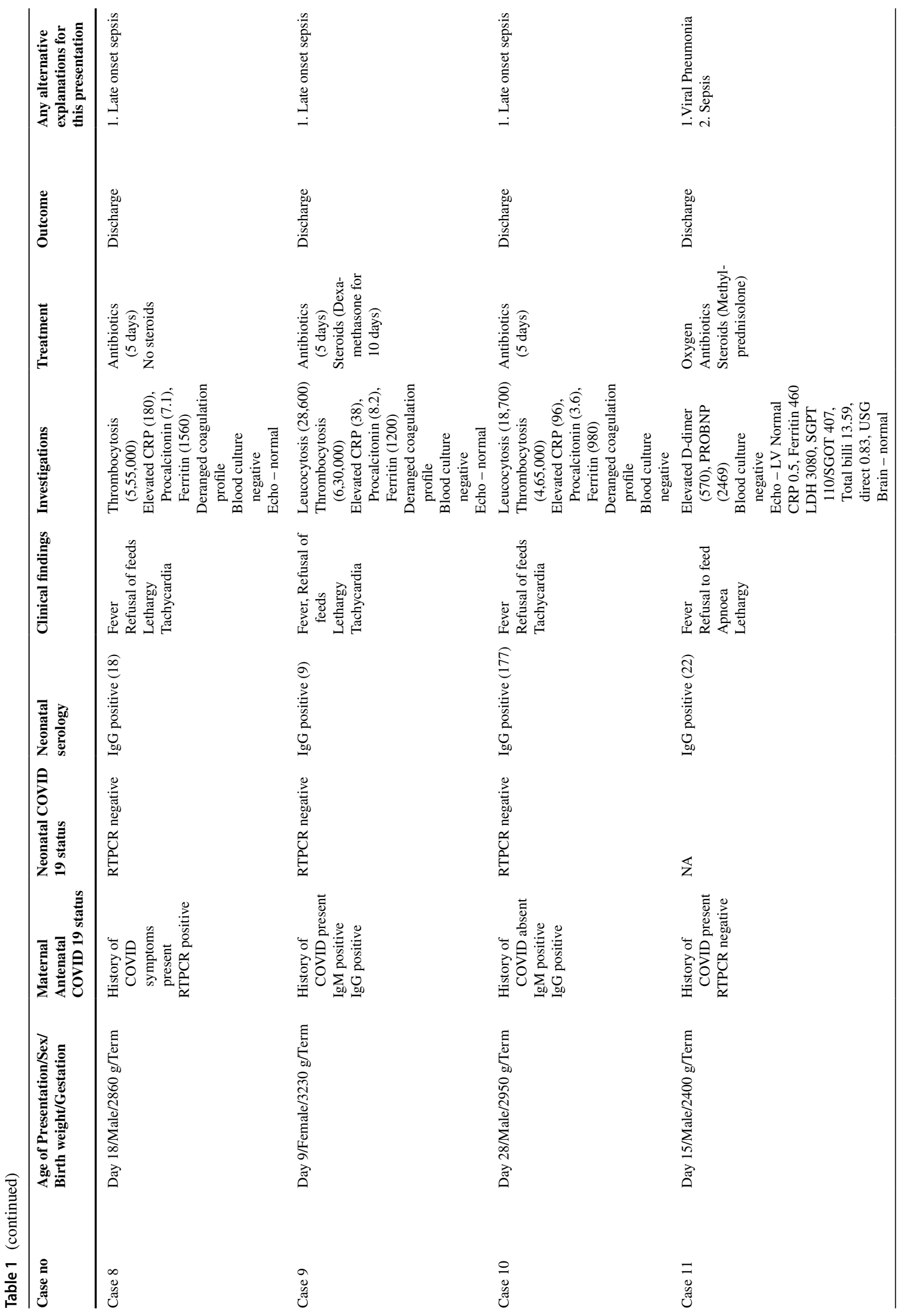




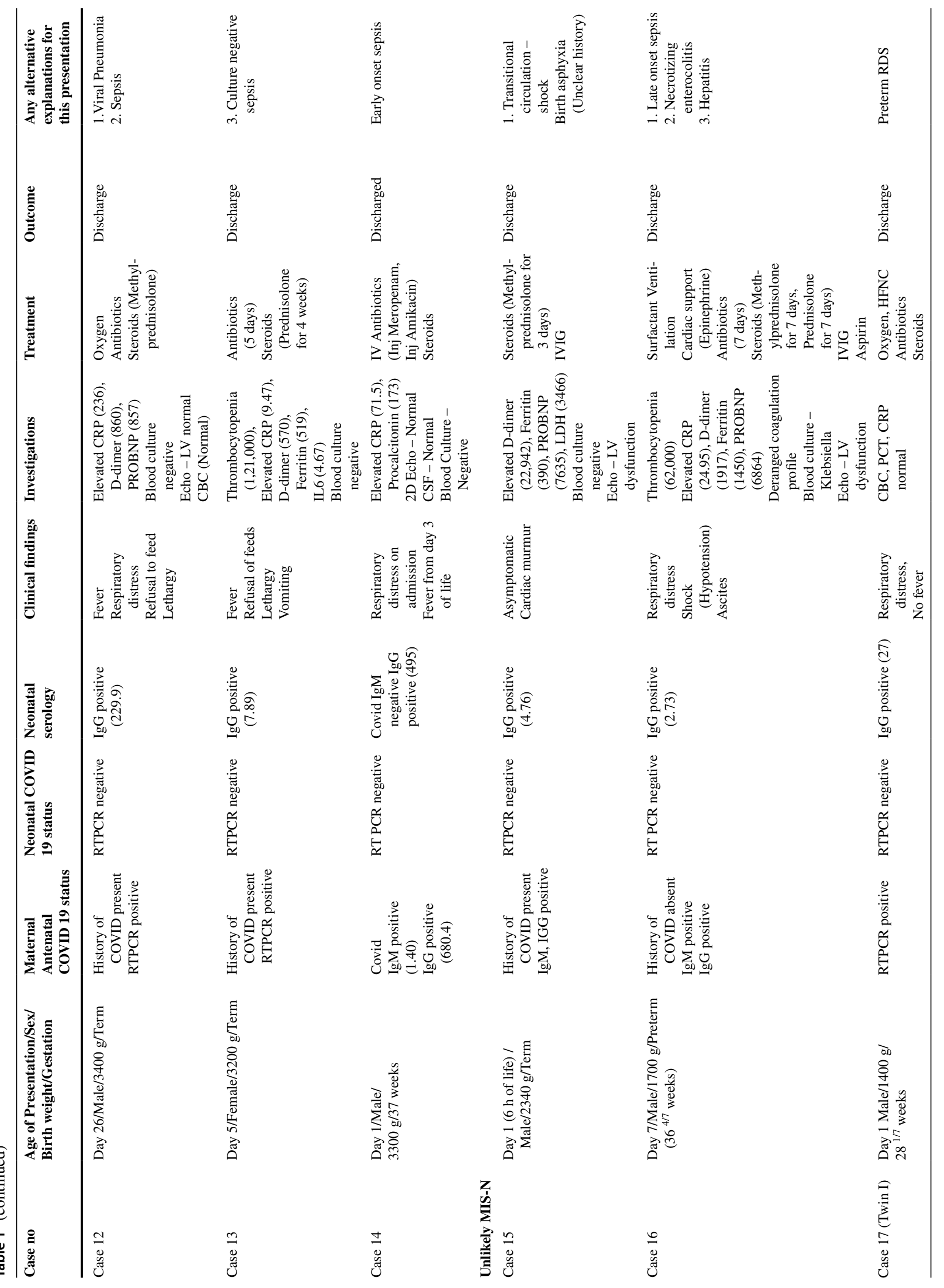




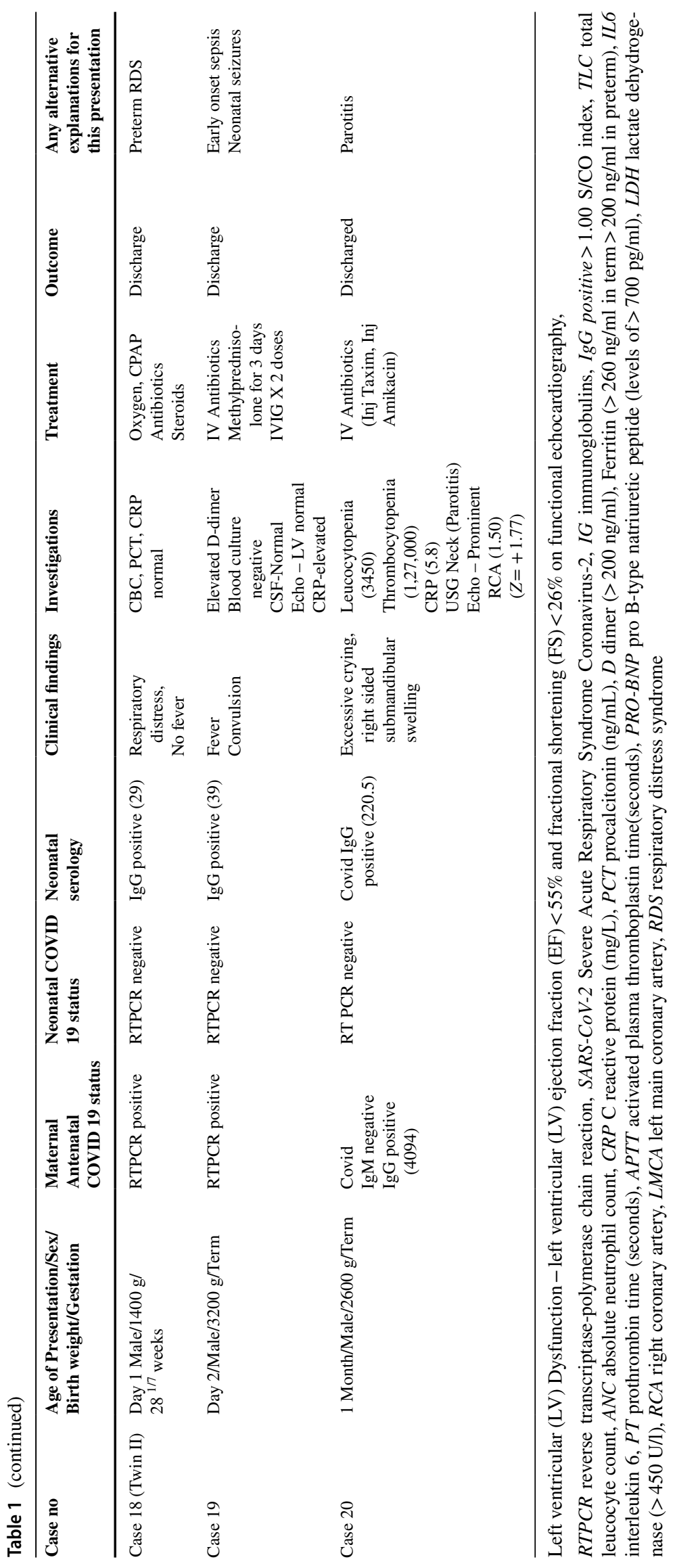


Fig. 2 Summary of clinical and laboratory findings of Most likely MIS-N cases

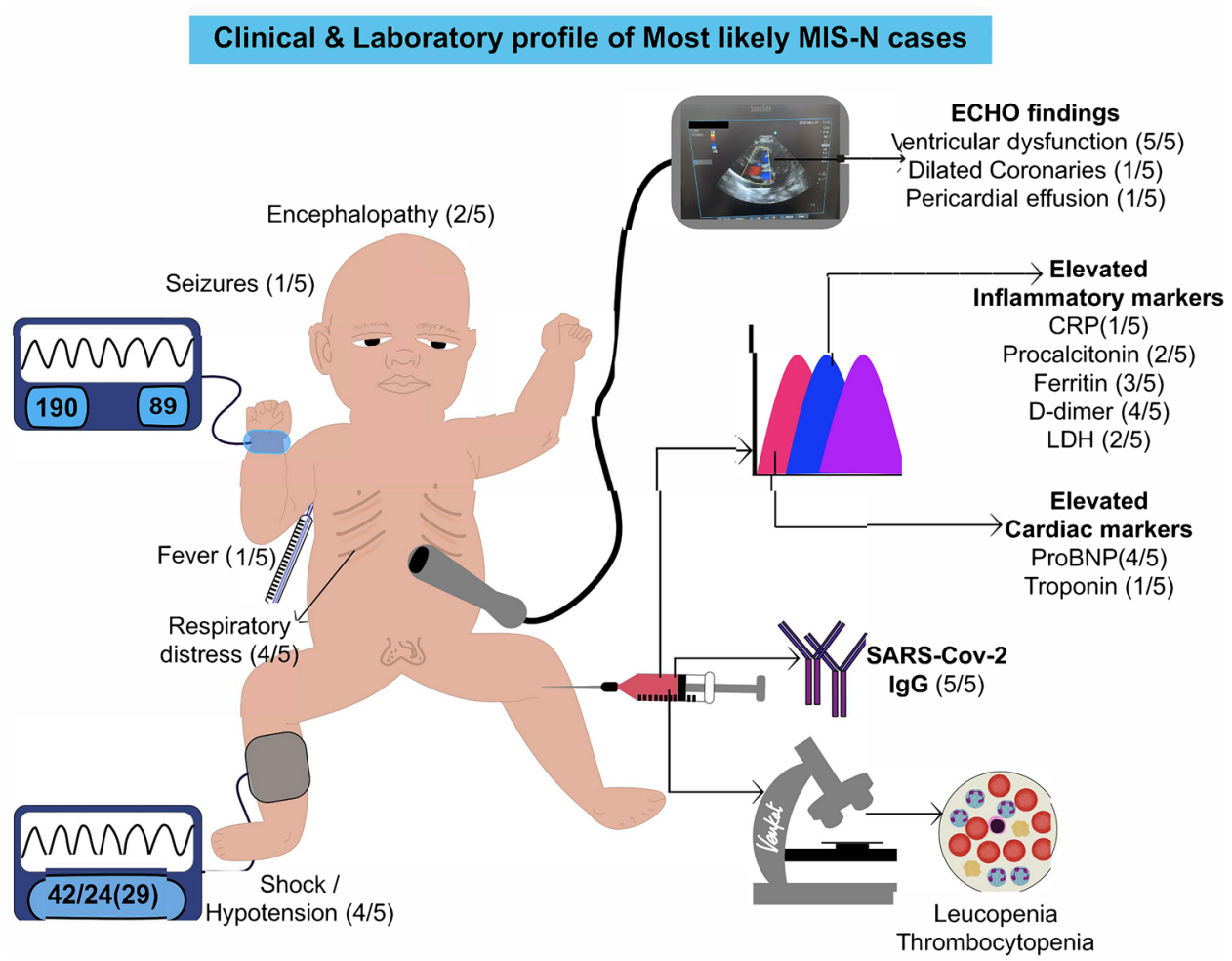

\section{Diagnostic assessment}

All 20 neonates had positive serology in the form of high titres of SARS CoV 2 IgG antibodies and negative SARS $\mathrm{CoV} 2$ antigen during the presentation of MIS. SARS CoV $2 \mathrm{IgG}$ antibodies were positive in $8 / 20$ mothers, whereas SARS CoV 2 antigen was positive on RTPCR in 9/10. History of previous maternal SARS CoV 2 infection was presents in remaining 10.

Most likely MIS Inflammatory markers were found to be elevated in these neonates with raised CRP (1/5), Procalcitonin (1/5), Ferritin (3/5), D-dimer (4/5), and LDH (2/5). In addition, four of them had significantly high levels of proBNP, and one had high levels of Troponin T. All of them had cardiac dysfunction on echo, and only one neonate had dilated coronaries with pericardial effusion.

Possible MIS Elevated CRP was documented in five neonates and procalcitonin (PCT) in three infants. The inflammatory markers associated with SARS-CoV-2 infection were markedly elevated D-dimer (6/9) and Ferritin (5/9). Interleukin- 6 was measured and found to be elevated in one infant, whereas ESR levels were not measured in any patient. ProBNP as a marker of cardiac dysfunction was noted to be elevated in five infants. Haematological abnormalities noted in this cohort were leucocytosis in two, thrombocytopenia in two, and thrombocytosis in three infants. Echocardiography was performed in seven neonates, two of them had cardiac dysfunction, and one had PPHN. Coronary vessels were found to be borderline abnormal in only one.

Unlikely MIS These newborns also had elevated inflammatory markers, with one newborn having LV dysfunction and significantly elevated ProBNP.

\section{Therapeutic interventions}

Most likely MIS The majority of them (4/5) required immunomodulators in the form of steroids and IVIG. All the neonates required respiratory support in the form of oxygen or ventilation and cardiac support in the form of inotropes.

Possible MIS Immunomodulators were given to seven infants in the form of steroids (5/7) or steroids along with IVIG (2/7).

Unlikely MIS The majority of newborns in this group were also treated with immunomodulators, mainly steroids(5/6), and 2/6 were also additionally treated with IVIG.

\section{Outcome}

In the Most likely MIS group, three infants were discharged home, and two neonates succumbed to the illness. All infants in the Possible and Unlikely MIS groups were discharged home. 
Using medical records all the patient families were chased telephonically to obtain follow up information at 6 to 12 months of age (variable due to the different time points that they presented at). Out of 18 survivors, clinicians had no concerns with respect to the development or long-term morbidities for any of them except for two who were lost to follow up.

Comparative analysis We compared clinical presentation and outcomes of all these 20 cases based on the timing of presentation correlating with the possible mechanism of MIS-N. The summary of this comparison is enlisted in Table 2.

Ten cases presented early within $72 \mathrm{~h}$, whereas the remaining 10 presented beyond $72 \mathrm{~h}$. Preterm infants tend to present early compared to term infants $(p<0.01)$. Respiratory distress $(70 \%)$ and cardiac dysfunction $(60 \%)$ tend to be more common during the early presentation ( $p$-value not significant), whereas fever (70\%) and sepsis-like symptoms (lethargy, poor feeding) (60\%) tends to be more common in late presentation $(p=0.02)$. There was no difference in investigations, management, or outcomes between the two groups.

\section{Discussion}

MIS-C in the paediatric population presents a new challenge in the COVID-19 pandemic, with few cases reported worldwide [27]. As MIS-C generally occurs after a lag period following SARS-CoV-2 infection, correlating with the timing of developing anti-spike antibodies (IgG), it is hypothesised to be caused by aberrant cellular or humoral adaptive immune responses triggering inflammation or mediating organ damage [3, 27, 28]. In newborns, SARS-CoV-2 infection can present either as an early-onset infection, likely due to vertical or intrapartum transmission [29] of SARS-CoV-2 or late-onset SARS-CoV-2 infection, more than $72 \mathrm{~h}$ after birth, which may be acquired through close contacts [21]. Transplacental transmission of SARS-CoV-2 is less common in term babies due to lack of expression of angiotensinconverting enzyme (ACE) 2 and transmembrane protease serine 2 (TMPRSS2) by trophoblasts, which are two main host membrane receptors for SARS-CoV-2 entry; however, this raises concerns in preterm [30]. Maternal SARS-CoV2 infection results in the development of protective ant-spike IgG antibodies which cross the placenta and may offer protection to newborns along with secretory IG-A which are secreted in breast milk [31]. However, it is postulated that in certain genetically susceptible newborns, these antibodies may bind to receptors on neutrophils and macrophages causing activation of cytokines leading to various manifestations of MIS-N. It is also postulated that some newborns may develop MIS-N due to a mechanism similar to MIS-C, caused by autoantibody-mediated immune dysregulation in response to perinatally acquired SARS-CoV2 infection [30] (Fig. 3).

It was speculated that neonates might have less inflammation, milder COVID-19 illness, and faster recovery compared to older children and adults due to passive transfer of protective maternal IgG antibodies, immature immune system, presence of fetal haemoglobin, and lower angiotensinconverting enzyme-2 (ACE-2) expression [32, 33]. MIS-N is emerging as a new disease with a few cases being reported globally [34]. We report a case series of a variety of clinical manifestations of MIS-N, which is hypothesised to be caused by either transplacental transfer of SARS-CoV2 antibodies or antibodies developed in the neonate after infection with SARS-CoV-2.

The diagnosis of SARS-CoV-2 infection in neonates due to vertical transmission not only requires positive SARS$\mathrm{CoV} 2$ antigen in respiratory secretions for both the mother and neonate but also demonstration of the virus in amniotic fluid/placenta and cord blood along with the presentation of neonatal disease attributable to COVID-19 [35]. Diagnosing MIS-N in neonates is even more challenging. MIS-N is proposed to be a disease manifestation of antibody-mediated immune activation affecting various organs rather than the infection itself [3]. Hence, to differentiate neonatal SARSCoV2 infection from MIS-N, the neonate should be negative for SARS-CoV2 antigen but positive for SARS-CoV2 antibodies, either trans-placentally transmitted from the mother or produced by self after an interval of recovery from SARS-CoV2 infection. Moreover, the clinical manifestations of MIS-N are very similar to sepsis-like illnesses and other conditions associated with prematurity, making it difficult to differentiate between them on clinical grounds alone.

In our cohort, we scrutinised the cases that were treated as MIS-N by clinicians and categorised them into Most likely and Possible MIS on the basis of pre-defined clinical criteria and also reviewed indications for commencing immunomodulatory therapies. Cases that were treated as MIS by their clinicians but did not fit specified criteria and had other possible explanations for the clinical presentation were classified as Unlikely MIS. We specifically included these cases to highlight how the presence of antibodies and some of the biochemical parameters can be confusing, leading to a risk of overdiagnosis and potentially incorrect treatment during the pandemic.

In our cohort, preterm infants tend to present early compared to term infants with the more severe disease commonly presenting as respiratory distress and cardiac dysfunction, similar to the observation by Lima et al. [10]. Fever was a striking feature in late presenting neonates and in term neonates leading to suspicion of possible postnatally acquired MIS-N, but skin manifestations and gastrointestinal symptoms were not common, in contrast 
Table 2 Clinical parameters and outcomes based on timing of presentation

\begin{tabular}{|c|c|c|c|c|}
\hline & Clinical parameters and outcomes & Early MISN (within 72 h) & Late MISN (beyond $72 \mathrm{~h}$ ) & $P$ value \\
\hline 1 & Number of cases & $10 / 20(50 \%)$ & $10 / 20(50 \%)$ & 1 \\
\hline \multicolumn{5}{|c|}{ Term $(\mathrm{T})>37$ weeks } \\
\hline 3 & Age of presentation & $\begin{array}{l}\text { Day } 1=8(80 \%) \\
\text { Day } 2=2(20 \%)\end{array}$ & $\begin{aligned} \text { Week } 1 & =3 \\
\text { Week } 2 & =1 \\
\text { Week } 3 & =2 \\
\text { Week } 4 & =4\end{aligned}$ & - \\
\hline \multirow[t]{10}{*}{4} & \multicolumn{4}{|l|}{ Clinical Presentation } \\
\hline & RDS & $7(70 \%)$ & $4(40 \%)$ & 0.17 \\
\hline & Cardiac dysfunction & $6(60 \%)$ & $3(30 \%)$ & 0.18 \\
\hline & PPHN & $2(20 \%)$ & 0 & - \\
\hline & Fever & $2(20 \%)$ & $7(70 \%)$ & 0.02 \\
\hline & Seizures & $2(20 \%)$ & 0 & - \\
\hline & Encephalopathy & $1(10 \%)$ & 0 & - \\
\hline & Sepsis like & $1(10 \%)$ & $6(60 \%)$ & 0.02 \\
\hline & Hypoglycemia & 0 & $1(10 \%)$ & - \\
\hline & Parotitis & 0 & $1(10 \%)$ & - \\
\hline \multirow[t]{15}{*}{5} & \multicolumn{4}{|l|}{ Abnormal Lab parameters: } \\
\hline & Inflammatory: CRP & $3(30 \%)$ & $7(70 \%)$ & 0.08 \\
\hline & PCT & $2(20 \%)$ & $4(40 \%)$ & 0.34 \\
\hline & D-Dimer & $7(70 \%)$ & $5(50 \%)$ & 0.37 \\
\hline & Ferritin & $3(30 \%)$ & $7(70 \%)$ & 0.08 \\
\hline & \multicolumn{4}{|l|}{ Cardiac biomarkers: } \\
\hline & $\mathrm{BNP}$ & $6(60 \%)$ & $4(40 \%)$ & 0.38 \\
\hline & TropT & 0 & $1(10 \%)$ & - \\
\hline & $\mathrm{LDH}$ & $3(30 \%)$ & $2(20 \%)$ & 0.61 \\
\hline & Hematological: & & & - \\
\hline & Leukocytosis & 0 & $2(20 \%)$ & 1 \\
\hline & Leukocytopenia & $1(10 \%)$ & $1(10 \%)$ & 0.27 \\
\hline & Thrombocytopenia & $1(10 \%)$ & $3(30 \%)$ & - \\
\hline & Thrombocytosis & 0 & $3(30 \%)$ & 0.27 \\
\hline & Coagulation & $1(10 \%)$ & $3(30 \%)$ & \\
\hline \multirow[t]{5}{*}{6} & \multicolumn{4}{|l|}{ ECHO parameters } \\
\hline & LV dysfunction & $5(50 \%)$ & $2(20 \%)$ & 0.17 \\
\hline & Dilated coronaries & 0 & $2(20 \%)$ & - \\
\hline & PPHN & $2(20 \%)$ & $1(10 \%)$ & 0.54 \\
\hline & Pericardial effusion & 0 & $1(10 \%)$ & - \\
\hline \multirow[t]{13}{*}{7} & \multicolumn{4}{|l|}{ Management } \\
\hline & \multicolumn{4}{|l|}{ Respiratory support: } \\
\hline & Oxygen & $1(10 \%)$ & $2(20 \%)$ & 0.54 \\
\hline & Non-invasive & $2(20 \%)$ & $1(10 \%)$ & 0.54 \\
\hline & Ventilation & $4(40 \%)$ & $1(10 \%)$ & 0.13 \\
\hline & \multicolumn{4}{|l|}{ Cardiac support: } \\
\hline & Inotropes & $5(50 \%)$ & $2(20 \%)$ & 0.17 \\
\hline & Sildenafil & $1(10 \%)$ & 0 & - \\
\hline & \multicolumn{4}{|l|}{ Immunomodulator therapy: } \\
\hline & Steroids & $10(100 \%)$ & $7(70 \%)$ & 0.06 \\
\hline & IVIG & $6(60 \%)$ & $2(20 \%)$ & 0.07 \\
\hline & Aspirin & $1(10 \%)$ & $2(20 \%)$ & 0.54 \\
\hline & Antibacterial therapy: & $10(100 \%)$ & $10(100 \%)$ & 1 \\
\hline
\end{tabular}


Table 2 (continued)

\begin{tabular}{|c|c|c|c|c|}
\hline & Clinical parameters and outcomes & Early MISN (within 72 h) & Late MISN (beyond 72 h) & $P$ value \\
\hline \multirow[t]{3}{*}{8} & Outcome: & & & \\
\hline & Death & $1(10 \%)$ & $1(10 \%)$ & 1 \\
\hline & Discharge home & $9(90 \%)$ & $9(90 \%)$ & \\
\hline
\end{tabular}

to frequent reports in older children [7, 36]. Similar to the MIS-N case reported by Borkotoky et al. [12], one of the preterm infants, born at 34 weeks (case 3), presented at birth with manifestations of severe cytokine storm, with features of severe myocardial dysfunction, severe pulmonary hypertension, and elevated biomarkers suggesting severe disease. We observed, in addition, a few term babies also presenting with cardiac dysfunction and severe multisystem disease.

Majority of the newborns in the cohort especially Most likely and Probable MIS demonstrated elevations of inflammatory markers. Although Ferritin and D-Dimer are acute phase reactants and levels may be elevated in sepsis-like illnesses, a diagnosis of MIS-N was made in the clinical
Fig. 3 Proposed pathogenesis of MIS-N

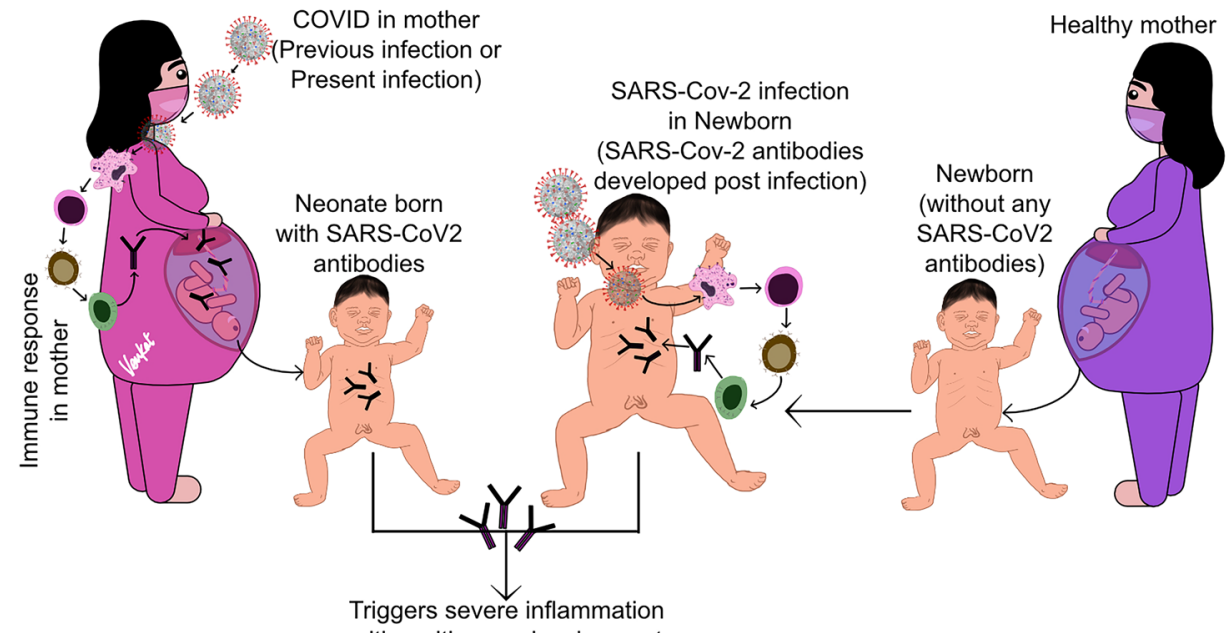

inflammation with multi-organ involvement

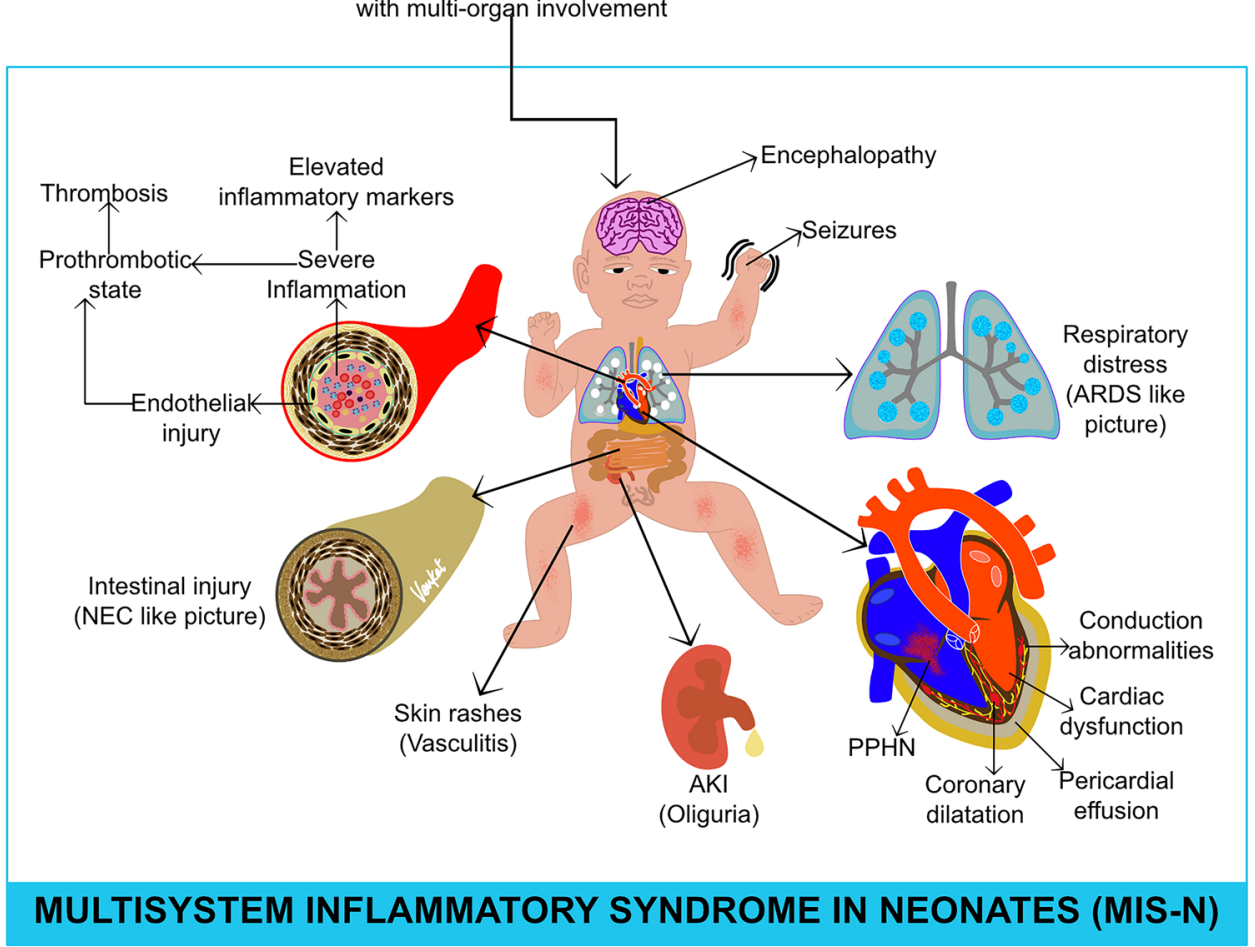


context with other elevated relevant markers and negative blood cultures. Moreover, ProBNP, as a marker of cardiac dysfunction, was elevated significantly in all neonates with cardiac dysfunction, suggesting immune-mediated myocarditis, similar to a case report of a 24-day-old neonate by Kappanayil et al. [11]. Cardiac biomarker, ProBNP, can be raised in premature infants [37] with patent ductus arteriosus, but levels noted in this preterm cohort were exponentially high, suggesting myocarditis. Troponin $\mathrm{T}$ was done in only one case and was elevated, supporting the diagnosis. ProBNP and Troponin may be helpful as early predictors of cardiac dysfunction in MIS-N; this, however, needs further study in larger cohorts. We did not find coronary aneurysm in any patient as reported in the paediatric population [26] except one baby who had marginally dilated coronaries. There was also no report of cardiac arrhythmia similar to that reported in a published neonatal cohort [5].

SARS-CoV2 IgG antibodies were positive in all, suggesting possible transplacental transmission of IgG. However, we could not confirm this in some infants presenting late who may have developed these antibodies due to perinatally acquired infection.

Clinicians managing our cohort of MIS-N cases used steroids (methylprednisolone or prednisolone) either alone or in combination with IVIG as per recommendations by ICMR 2020 (Appendix). In the term group, fever in two babies responded only after commencing steroids. In the severely affected infants with associated cardiac dysfunction, most babies showed improvement in clinical and biochemical parameters after both steroids and IV IG administration. However, the use of immunomodulator therapy in unlikely MIS cohort may not be justified, especially in the presence of possible other diagnoses and thus clinicians should refrain from routine use of steroids in such cases. All neonates in our cohort, except two, recovered completely, with no need for prolonged ventilation or extracorporeal membrane oxygenation (ECMO). Generally, environmentally acquired SARS$\mathrm{CoV} 2$ infection in newborns seems to be a more severe disease [21]; however, we did not find any significant difference in morbidity or outcomes between early and late presenters.

In the current rapidly spreading pandemic, we would like to caution clinicians regarding the increasing number of pregnant mothers who are either exposed to SARS-CoV-2 during pregnancy or have received a COVID vaccine or, less commonly, may have acquired antibodies via transfusion of any blood product. Transfer of these antibodies to the newborn may or may not cause MIS-N in the infant. It could however result in overdiagnosis of MIS by treating clinicians if these antibodies are incidentally detected during another illness. Thus, typing antibodies for anti-nucleoprotein antibodies suggesting past infection rather than anti-spike protein antibodies suggesting vaccination status can be considered to avoid confusion in situations where there is no definite evidence of present and past maternal SARS-CoV2 infection.

\section{Limitations}

This is a small case series with self-reported data and therefore has its limitations. However, various clinical presentations are described, and this preliminary data may add to the growing evidence on MIS-N. Not all currently recommended parameters and inflammatory markers, including COVID testing of infants at birth, were uniformly tested in all patients, leading to difficulty in confirming the diagnosis in some cases. There was also no routine testing for other viruses, and therefore, we cannot rule out other viral illnesses with certainty, which may present and respond to treatment similarly. This may be because MIS-N is still evolving as a disease entity with no specific clear guidance on diagnosis and management, combined with a general lack of awareness amongst clinicians.

\section{Conclusions}

MIS-N presents as a syndrome occurring post-exposure to SARS-CoV2, manifesting with a variety of clinical presentations. The diagnosis of MIS-N can be challenging and needs a high index of suspicion, after all other potential aetiologies have been ruled out. MIS-N can be considered in a neonate presenting with two or more systems involvement, in the presence of SARS-CoV2 antibodies, along with elevated inflammatory markers. Where suspected, neonatologists should perform specific investigations such as SARS-CoV2 antigen and antibodies, inflammatory markers routinely along with cardiac biomarkers like ProBNP, LDH, Troponin, and early ECHO screening to assess myocardial dysfunction when indicated. Immunomodulator therapy should be reserved for severe cases only where definitive criteria of MIS-N are met. There is a need for a more systematic process of registration of cases nationally and clear management guidelines for suspected MIS-N. Further studies are required to identify risk factors for MIS-N and also predictors of disease severity.

Supplementary information The online version contains supplementary material available at https://doi.org/10.1007/s00431-022-04377-z.

Acknowledgements Dr Nawaz Patel, Dr Hitesh Desai, and Dr Shwetal Bhatt for contributing to data collection. Parents of the patients included in this cohort who provided written informed consent for publication and also respective departmental heads for giving permission to share their data. 
Authors' contributions Dr Kiran More, Dr. Roopali Soni, and Dr Praveen Kumar conceptualised and designed the study, coordinated and supervised data collection, drafted the initial manuscript, and reviewed and revised the final manuscript. Dr Kiran and Dr Roopali revised the revisions of manuscript. Dr. Sheila Aiyer involved in design of study; obtained local ethics committee approval; contributed, coordinated, and supervised data collection; and reviewed/approved initial and final manuscript. Dr. Ashish Goti, Dr. Manan Parikh, Dr. Samir Sheikh, and Dr. Gaurav Patel all involved in design of the study; they contributed cases, coordinated data collection, and carried out the initial analyses; critically reviewed the manuscript; and approved final manuscript. Dr. Venkat Kallem created figures, helped out detailed analysis, formatted tables, and reviewed, edited, and approved the initial and final draft of the manuscript.

\section{Availability of data and material Not applicable.}

Code availability Not applicable.

\section{Declarations}

Ethics approval Ethics committee approval was obtained from the primary site SSG Medical College and Hospital, Vadodara, Gujarat.

Consent to participate Parental consent was obtained for each case where data was shared, along with permission from local hospital authorities for data sharing.

Consent for publication We consent for publishing data or images from our study for increasing awareness for this condition.

Conflict of interest The authors declare no competing interests.

\section{References}

1. Al Ameer HH, AlKadhem SM, Busaleh F, AlKhwaitm S, Llaguno MBB (2020) Multisystem inflammatory syndrome in children temporally related to COVID-19: a case report from Saudi Arabia. Cureus 12(9):e10589

2. Radia T, Williams N, Agrawal P, Harman K, Weale J, Cook J et al (2020) Multi-system inflammatory syndrome in children \& adolescents (MIS-C): a systematic review of clinical features and presentation. Paediatr Respir Rev

3. Nakra NA, Blumberg DA, Herrera-Guerra A, Lakshminrusimha S (2020) Multi-System Inflammatory Syndrome in Children (MISC) following SARS-CoV-2 infection: review of clinical presentation, hypothetical pathogenesis, and proposed management. Children (Basel) 7(7)

4. Flannery DD, Gouma S, Dhudasia MB, Mukhopadhyay S, Pfeifer MR, Woodford EC et al (2021) Assessment of maternal and neonatal cord blood SARS-CoV-2 antibodies and placental transfer ratios. JAMA Pediatr

5. Pawar R, Gavade V, Patil N, Mali V, Girwalkar A, Tarkasband V et al (2021) Neonatal Multisystem Inflammatory Syndrome (MIS$\mathrm{N})$ associated with prenatal maternal SARS-CoV-2: a case series. Children 8(7):572

6. Long QX, Liu BZ, Deng HJ, Wu GC, Deng K, Chen YK et al (2020) Antibody responses to SARS-CoV-2 in patients with COVID-19. Nat Med 26(6):845-848

7. Orlanski-Meyer E, Yogev D, Auerbach A et al (2020) Multisystem Inflammatory Syndrome in Children associated with severe acute respiratory syndrome coronavirus-2 in an 8-week-old infant. J Pediatric Infect Dis Soc 9(6):781-784
8. Abrams JY, Oster ME, Godfred-Cato SE, Bryant B, Datta SD, Campbell AP et al (2021) Factors linked to severe outcomes in multisystem inflammatory syndrome in children (MIS-C) in the USA: a retrospective surveillance study. Lancet Child Adolesc Health 5(5):323-331

9. Dufort EM, Koumans EH, Chow EJ, Rosenthal EM, Muse A, Rowlands J et al (2020) Multisystem Inflammatory Syndrome in Children in New York State. N Engl J Med 383(4):347-358

10. Lima ARO, Cardoso CC, Bentim PRB, Voloch CM, Rossi ÁD, da Costa RMMSC et al (2021) Maternal SARS-CoV-2 infection associated to systemic inflammatory response and pericardial effusion in the newborn: a case report. J Pediatric Infect Dis Soc 10(4):536-539. https://doi.org/10.1093/jpids/piaa133 (PMID: 33125068; PMCID: PMC7665586)

11. Kappanayil M, Balan S, Alawani S et al (2021) Multisystem inflammatory syndrome in a neonate, temporally associated with prenatal exposure to SARS-CoV-2: a case report. Lancet Child Adolesc Health 5(4):304-308

12. Khaund Borkotoky R, Banerjee Barua P, Paul SP, Heaton PA (2021) COVID-19-related potential multisystem inflammatory syndrome in childhood in a neonate presenting as persistent pulmonary hypertension of the newborn. Pediatr Infect Dis J

13. Divekar AA, Patamasucon P, Benjamin JS (2021) Presumptive neonatal multisystem inflammatory syndrome in children associated with coronavirus disease 2019. Am J Perinatol 38:632-636

14. Shaiba LA, Hadid A, Altirkawi KA et al (2021) Case report: Neonatal multi-system inflammatory syndrome associated with SARS-CoV-2 exposure in two cases from Saudi Arabia. Front Pediatr 13(9):652857. https://doi.org/10.3389/fped.2021.652857

15. Diggikar S, Nanjegowda R, Kumar A, Kumar V, Kulkarni S, Venkatagiri P (2021) Neonatal Multisystem Inflammatory Syndrome secondary to SARS-CoV-2 infection. J Paediatr Child Health 13. https://doi.org/10.1111/jpc.15696

16. Diwakar K, Gupta BK, Uddin MW, Sharma A, Jhajra S (2021) Multisystem inflammatory syndrome with persistent neutropenia in neonate exposed to SARS-CoV-2 virus: A case report and review of literature. J Neonatal Perinatal Med. https://doi.org/10. 3233/NPM-210839

17. More K, Chawla D, Murki S, Tandur B, Deorari AK, Kumar P (2021) National Neonatology Forum (NNF) COVID-19 Registry Group. Outcomes of neonates born to mothers with coronavirus disease 2019 (COVID-19) — National Neonatology Forum (NNF) India COVID-19 Registry. Indian Pediatr 58(6):525-531. https:// doi.org/10.1007/s13312-021-2234-2 (Epub 2021 Mar 20. PMID: 33742609; PMCID: PMC8253678)

18. Chawla D, Chirla D, Dalwai S, Deorari AK, Ganatra A, Gandhi A, Kabra NS, Kumar P, Mittal P, Parekh BJ, Sankar MJ, Singhal T, Sivanandan S, Tank P (2020) Federation of Obstetric and Gynaecological Societies of India (FOGSI), National Neonatology Forum of India (NNF) and Indian Academy of Pediatrics (IAP). Perinatal-Neonatal Management of COVID-19 Infection - Guidelines of the Federation of Obstetric and Gynaecological Societies of India (FOGSI), National Neonatology Forum of India (NNF), and Indian Academy of Pediatrics (IAP). Indian Pediatr 57(6):536-548. https://doi.org/10.1007/s13312-020-1852-4 (Epub 2020 Apr 1. PMID: 32238615; PMCID: PMC7340735)

19. MOHFW Clinical management protocol : COVID 19 Version 3: 13/06/2020. https://www.mohfw.gov.in/pdf/ClinicalManagem entProtocolforCOVID19.pdf

20. Centers for Disease Control and Prevention (2020) Multisystem inflammatory syndrome in children (MIS-C) associated with coronavirus disease 2019 (COVID-19). CDC Health Alert Network. https://www.cdc.gov/mis/about.html

21. Raschetti R, Vivanti AJ, Vauloup-Fellous C, Loi B, Benachi A, De Luca D (2020) Synthesis and systematic review of reported 
neonatal SARS-CoV-2 infections. Nat Commun 11(1):5164. https://doi.org/10.1038/s41467-020-18982-9

22. World Health Organization (2021) Definition and categorization of the timing of mother-to-child transmission of SARSCoV-2: scientific brief, 8 Feb 2021. World Health Organization. https:// www.who.int/publications/i/item/WHO-2019-nCoV-mother-tochild-transmission-2021.1

23. Siddappa AM, Rao R, Long JD, Widness JA, Georgieff MK (2007) The assessment of newborn iron stores at birth: a review of the literature and standards for ferritin concentrations. Neonatology 92(2):73-82

24. Hur M, Kim H, Lee S, Cristofano F, Magrini L, Marino R et al (2014) Diagnostic and prognostic utilities of multimarkers approach using procalcitonin, B-type natriuretic peptide, and neutrophil gelatinase-associated lipocalin in critically ill patients with suspected sepsis. BMC Infect Dis 14:224

25. Singh Y (2017) Echocardiographic evaluation of hemodynamics in neonates and children. Front Pediatr 5:201

26. Riley DS, Barber MS, Kienle GS, AronsonJK, von SchoenAngerer T, Tugwell P, Kiene H, Helfand M, Altman DG, Sox H, Werthmann PG, Moher D, Rison RA, Shamseer L, Koch CA, Sun GH, Hanaway P, Sudak NL, Kaszkin-Bettag M, Carpenter JE, Gagnier JJ (2017) CARE guidelines for case reports: explanation and elaboration document. J Clin Epidemiol S08954356(17)30037-9. https://doi.org/10.1016/j.jclinepi.2017.04.026

27. Levin M (2020) Childhood Multisystem Inflammatory Syndrome - a new challenge in the pandemic. N Engl J Med 383(4):393-395

28. Liu L, Wei Q, Lin Q et al (2019) Anti-spike IgG causes severe acute lung injury by skewing macrophage responses during acute SARS-CoV infection. JCI Insight 4(4):e123158

29. Vivanti AJ, Vauloup-Fellous C, Prevot S, Zupan V, Suffee C, Do Cao J, Benachi A, De Luca D (2020) Transplacental transmission of SARS-CoV-2 infection. Nat Commun 11(1):3572. https:// doi.org/10.1038/s41467-020-17436-6.PMID:32665677;PMCID: PMC7360599
30. Colson A, Depoix CL, Dessilly G, Baldin P, Danhaive O, Hubinont C, Sonveaux P, Debiève F (2021) Clinical and in vitro evidence against placenta infection at term by severe acute respiratory syndrome coronavirus 2. Am J Pathol 191(9):1610 1623. https://doi.org/10.1016/j.ajpath.2021.05.009 (Epub 2021 Jun 8. PMID: 34111431; PMCID: PMC8184362)

31. Sankaran D, Nakra N, Cheema R, Blumberg D, Lakshminrusimha S (2021) Perinatal SARS-CoV-2 infection and neonatal COVID19: a 2021 update. NeoReviews 22(5):e284-e295. https://doi.org/ 10.1542/neo.22-5-e1001 (PMID: 33931474)

32. Rawat M, Chandrasekharan $\mathrm{P}$, Hicar MD, Lakshminrusimha S (2020) COVID-19 in newborns and infants-low risk of severe disease: Silver Lining or Dark Cloud? Am J Perinatol 37(8):845-849

33. Zeng H, Xu C, Fan J et al (2020) Antibodies in infants born to mothers with COVID-19 pneumonia. JAMA 323(18):1848-1849

34. Sánchez-Luna M, Fernández Colomer B, de Alba Romero C et al (2021) Neonates born to mothers with COVID-19: data from the Spanish Society of Neonatology Registry. Pediatrics 147(2)

35. Blumberg DA, Underwood MA, Hedriana HL, Lakshminrusimha S (2020) Vertical transmission of SARS-CoV-2: what is the optimal definition? Am J Perinatol 37(8):769-772

36. Abrams JY, Godfred-Cato SE, Oster ME et al (2020) Multisystem Inflammatory Syndrome in Children associated with Severe Acute Respiratory Syndrome Coronavirus 2: a systematic review. J Pediatr 226:45-54.e41

37. Harris SL, More K, Dixon B et al (2018) Factors affecting N-terminal pro-B-type natriuretic peptide levels in preterm infants and use in the determination of the haemodynamic significance of patent ductus arteriosus. Eur J Pediatr 177(4):521-532

Publisher's Note Springer Nature remains neutral with regard to jurisdictional claims in published maps and institutional affiliations.

\section{Authors and Affiliations}

\section{Kiran More ${ }^{1}$ (]) Sheila Aiyer ${ }^{2} \cdot$ Ashish Goti $^{3} \cdot$ Manan Parikh $^{4} \cdot$ Samir Sheikh $^{5} \cdot$ Gaurav Patel $^{6} \cdot$ Venkat Kallem $^{7}$. Roopali Soni $^{1,8} \cdot$ Praveen Kumar $^{9}$}

\author{
Sheila Aiyer \\ sheilaaiyer@yahoo.com \\ Ashish Goti \\ drashishgoti@gmail.com \\ Manan Parikh \\ manan179@yahoo.com \\ Samir Sheikh \\ samirzuzma@yahoo.co.in \\ Gaurav Patel \\ drgauravpatel@yahoo.com \\ Venkat Kallem \\ venkat467@gmail.com \\ Roopali Soni \\ roopali.soni@gmail.com \\ Praveen Kumar \\ drpkumarpgi@gmail.com
}

1 Sidra Medicine Hospital, Education City North Campus, Qatar Foundation, Doha, Qatar

2 SSG Medical College and Hospital, Vadodara, Gujarat, India

3 Nice Children Hospital, Surat, Gujarat, India

4 Lotus Hospital for Newborn and Children, Vadodara, Gujarat, India

5 Wockhardt Hospitals, Mumbai, Maharashtra, India

6 Kashiben Govardhandas Patel Children's Hospital, Vadodara-Gujarat, India

7 Paramitha Children Hospital, Hyderabad, Telangana, India

Mediclinic Parkview Hospital, Dubai, UAE

9 Post Graduate Institute of Medical Education and Research, Chandigarh, India 